\title{
Les violences subies dans les différents espaces de vie en Martinique
}

Violence experienced in different areas of life in Martinique

\section{Elizabeth Brown et Nadine Lefaucheur}

\section{(2) OpenEdition}

Édition électronique

URL : http://journals.openedition.org/plc/863

DOI : $10.4000 /$ plc. 863

ISSN : 2117-5209

Éditeur

L'Harmattan

Édition imprimée

Date de publication : 1 janvier 2012

Pagination : 73-107

ISBN : 978-2-296-55-856-4

ISSN : 1279-8657

Référence électronique

Elizabeth Brown et Nadine Lefaucheur, «Les violences subies dans les différents espaces de vie en Martinique », Pouvoirs dans la Caraïbe [En ligne], 17 | 2012, mis en ligne le 26 janvier 2012, consulté le 19 avril 2019. URL : http://journals.openedition.org/plc/863 ; DOI : 10.4000/plc.863 


\title{
LES VIOLENCES SUBIES DANS LES DIFFÉRENTS ESPACES DE VIE EN MARTINIQUE
}

\author{
Elizabeth BROWN \\ Directrice du Centre de recherche de \\ l'Institut de démographie \\ Université Paris1 Panthéon-Sorbonne \\ Nadine LEFAUCHEUR \\ Sociologue \\ Membre associé du CRPLC \\ Université des Antilles et de la Guyane
}

Les personnes âgées de 18 à 59 ans, hommes et femmes vivant en Martinique depuis au moins un an et y ayant passé au moins neuf mois au cours de l'année écoulée, qui, jointes par téléphone, ont accepté de participer à l'enquête, ont d'abord répondu à de nombreuses questions d'ordre sociodémographique et biographique. Elles ont ensuite été interrogées sur les faits de violence subis au cours des douze mois écoulés, dans les sphères de la vie professionnelle, de la vie conjugale et des relations post-conjugales, des relations avec les proches (famille, amis, voisins) et dans les lieux et espaces publics. Elles ont finalement été interrogées sur les faits subis antérieurement, avant leur majorité, d'une part, depuis cette date, d'autre part.

\section{I. - LES VIOLENCES SUBIES AVANT DIX-HUIT ANS}

Parmi les violences déclarées pour les périodes antérieures aux douze derniers mois, les plus nombreuses et les plus graves se sont produites pendant l'enfance et l'adolescence des répondant(e)s : c'est le cas de deux tiers des situations d'humiliation et dénigrements systématiques déclarées par les hommes pour leur «vie entière » (douze derniers mois exclus) et de trois quarts de celles rapportées par les femmes, de neuf sur dix des brutalités relatées par les hommes et de six sur dix de celles qu'ont rapportées les femmes. C'est encore le cas des attouchements sexuels, dont neuf sur dix de ceux qui ont été rapportés ont eu lieu avant dix-huit ans, et enfin des tentatives de viol et des viols, surtout pour les garçons : s'ils s'en sont beaucoup moins souvent déclarés victimes que les filles, c'est, en revanche, beaucoup plus souvent pour la période où ils étaient mineurs (sept dixièmes des tentatives de viol déclarées et quatre cinquièmes des viols). 
Tableau 1 : Taux (en \%) d'atteintes et violences subies avant 18 ans selon le sexe

\begin{tabular}{|l|c|c|}
\hline \multicolumn{1}{|c|}{ Type de violence déclarée } & Femmes & Hommes \\
\hline Humiliations et dénigrement systématiques & 10,7 & 8,6 \\
\hline Coups violents ou brutalités & 5,9 & 8,2 \\
\hline Menaces de mort et tentatives de meurtre & 1,7 & 2,4 \\
\hline Attouchements forcés subis ou pratiqués & 7,5 & 1,5 \\
\hline Tentatives de viol & 3,5 & $<2 *$ \\
\hline Viols & 3,2 & $<1 *$ \\
\hline$*$ effectifs observés inférieurs à 10 & & \\
\hline
\end{tabular}

Les femmes sont un peu plus nombreuses que les hommes à avoir déclaré des humiliations et dénigrements systématiques pendant l'enfance et la jeunesse (tableau 1). Les seconds les situent nettement plus souvent dans le cadre scolaire et dans celui des relations d'amitié ou de voisinage que les premières, qui évoquent surtout les relations familiales et pour lesquelles le cumul ou la répétition des faits sont plus importants. Les hommes disent ainsi avoir été surtout humiliés par des camarades garçons et les femmes par des femmes de la famille, principalement leur mère - surtout lorsqu'elle était au foyer, inactive ou chômeuse. Plus d'un homme sur trois et près de trois femmes sur quatre estiment que ces humiliations et dénigrements ont eu des conséquences graves sur leur équilibre psychologique, voire sur leur vie en général.

Les hommes ont, en revanche, déclaré plus souvent avoir été violemment frappés ou brutalisés dans leur enfance ou leur adolescence (tableau 1) ; plus d'un pour cent en ont même été gravement blessés. Pour la moitié des garçons et quatre filles sur cinq, c'était dans le cadre familial. Pour les premiers, c'était deux fois sur trois par un homme de la famille, le père le plus souvent ; pour les secondes, c'était également le plus souvent le père ou le beau-père, voire un frère, mais c'était également souvent la mère ou une autre femme de la famille. Un quart des personnes qui ont été frappées violemment dans le cadre familial l'ont été par plusieurs personnes. Près d'un homme sur trois lie les humiliations ou les coups reçus à son origine ou à sa couleur, contre seulement une femme sur six.

La littérature comme les entretiens que nous avons réalisés en face-à-face témoignent de la prégnance d'un mode d'éducation «traditionnel», autoritaire et «musclé », reposant largement sur les reproches, les punitions et les corrections physiques; lors de l'enquête téléphonique, $30 \%$ des 
hommes et $21 \%$ des femmes nés en Martinique (vs $11 \%$ des personnes nées ailleurs) se sont d'ailleurs déclarés d'accord avec l'assertion : «pour bien élever un enfant, il faut le corriger physiquement ». Si près d'une personne sur cinq a répondu avoir «souvent été punie ou frappée injustement » dans son enfance ou son adolescence, nombre de celles qui ont donné une réponse négative l'ont ainsi commentée : «J'ai été souvent punie ou frappée, mais ce n'était pas injuste, puisque c'était pour mon bien ».

Il n'en reste pas moins que les menaces de mort ou les tentatives de meurtre avant dix-huit ans déclarées lors de l'enquête téléphonique (tableau 1) ou lors des témoignages recueillis dans les enquêtes qualitatives ne peuvent être imputés à un style éducatif « autoritaire mais juste». Pour la plupart, surtout pour les femmes, ces agressions ont eu lieu dans le cadre familial et ont été imputées soit à leur père ou beau-père, soit à leur mère ou belle-mère.

A l'instar des résultats établis pour la France hexagonale ${ }^{1}$, les femmes de Martinique ont davantage fait état d'atteintes et agressions sexuelles dans leur prime jeunesse que les hommes. Elles ont été cinq fois plus souvent qu'eux victimes d'attouchements sexuels, deux fois plus souvent de tentatives de viols et quatre fois plus souvent de viols (tableau 1). Six femmes sur dix situent les attouchements dans le cadre familial, les auteurs les plus fréquents étant un oncle, le père ou le beau-père, alors que deux sur dix citent un ami ou un voisin. Pour les garçons, les attouchements déclarés se sont moins souvent passés dans le cadre familial que dans celui des études ou des loisirs. Les tentatives déclarées par des hommes n'impliquaient pas de membres de la famille, mais un supérieur, des camarades et, dans un cas, plusieurs inconnus. En revanche, pour les trois hommes victimes de viols et pour trente des cinquante-deux femmes qui ont subi l'une et/ou l'autre de ces agressions, il s'agissait d'un membre de la famille : père, beau-père, oncle, frère, cousin, etc. ; six femmes ont été violées par leur petit ami et les autres ont été agressées par un camarade, un voisin ou des hommes qu'elles connaissaient (l'une désigne également une femme).

\section{II. - LES FACTEURS ASSOCIÉS AUX VIOLENCES SUBIES AVANT DIX-HUIT ANS}

Les principaux facteurs associés aux violences subies avant l'âge de dix-huit ans sont l'âge des personnes enquêtées, l'environnement familial qui était le leur dans l'enfance ou l'adolescence et le nombre de difficultés qu'elles ont déclaré avoir rencontré alors.

\footnotetext{
${ }^{1}$ Nathalie Bajos et Michel Bozon (dir). Enquête sur la sexualité en France : pratiques, genre et santé. Paris, La découverte, 2008. p. 388 et s.
} 
Quel que soit leur sexe, les répondant(e)s âgé(e)s de moins de 35 ans ont rapporté près de deux fois plus d'atteintes à leur dignité que les personnes plus âgées, tandis que les femmes de moins de 35 ans déclaraient plus d'agressions sexuelles que leurs aînées. Les personnes les plus jeunes ontelles réellement connu davantage ces deux types d'atteintes ou se sont-elles plus facilement remémoré une période de leur existence plus récente et moins «digérée », ou, encore, les plus âgées, ayant connu d'autres agressions au cours de leur vie adulte, ont-elles plutôt fait état de ces dernières, plus fraîches à leur mémoire ?

Comme on pouvait s'y attendre, on constate une corrélation extrêmement forte entre le nombre de difficultés vécues dans l'enfance ${ }^{2}$ et les violences subies avant dix-huit ans, quel qu'en soit le type. Des atteintes à la dignité ont été sept fois plus déclarées par les femmes et cinq fois plus par les hommes qui ont éprouvé au moins trois difficultés (avec des taux respectifs de $37 \%$ et $26 \%$ ) que par celles et ceux qui ont eu une enfance plus facile. Lorsqu'au moins trois difficultés ont été mentionnées, les taux de violences physiques sont multipliés par sept pour les hommes et par dix pour les femmes, et les taux d'agressions sexuelles sont quatre fois plus élevés pour les femmes.

En outre, l'environnement social et familial (approché par les questions portant sur la situation familiale et l'activité des parents lorsque les personnes enquêtées avaient quatorze ans) a sensiblement influencé les taux de victimation dans la jeunesse. Parmi les personnes dont la mère était au

\footnotetext{
${ }^{2}$ Les difficultés éprouvées pendant l'enfance ont été recensées par les neuf questions : «Avez-vous : souffert de la misère (pauvreté) ? manqué d'affection ? été souvent frappé(e) ou puni(e) injustement ? été maltraité(e) ou servi de souffre-douleur? eu un conflit très grave avec vos parents? vécu dans un climat de graves tensions ou de violences familiales? souffert de l'alcoolisme de l'un de vos proches ? Vous êtes-vous senti(e) rejeté(e) ou moins aimé(e) à cause de votre apparence physique ? Avez-vous été confié(e) pendant plus d'un an à un autre membre de votre famille que vos parents ou placé(e) dans une famille d'accueil ou une institution?».

$55 \%$ des hommes et $50 \%$ des femmes ont répondu négativement aux neuf questions. $21 \%$ des enquêté(e)s ont répondu positivement à un seul item; 17 à $18 \%$ ont répondu positivement à deux ou trois items et $11 \%$ des femmes et $6 \%$ des hommes ont fait état d'au moins quatre difficultés sur les neuf proposées.

Les deux questions qui ont obtenu le plus de réponses positives sont le manque d'affection et le fait d'avoir été souvent puni ou frappé injustement, mais les femmes ont mis en tête le manque d'affection tandis que les hommes citaient avant tout les coups et punitions injustes. Pour les hommes comme pour les femmes, la grande pauvreté vient en troisième position et le fait d'avoir été confié(e) pendant au moins un an à un membre de la famille autre que les parents ou placé(e) en famille d'accueil ou en institution en quatrième position. Outre le manque d'affection, ce sont les items portant sur le climat de tension ou de violence dans la famille, l'alcoolisme d'un proche, la maltraitance (ou le fait d'avoir «servi de souffredouleur ») et le sentiment d'avoir été rejeté(e)s ou moins aimé(e)s à cause de leur apparence physique auxquels les femmes ont plus particulièrement répondu positivement.
} 
foyer, chômeuse ou inactive, les femmes ont ainsi déclaré plus d'humiliations et de dénigrements que les autres, et les hommes plus d'atteintes sexuelles. Ceux dont le père travaillait ont davantage évité les insultes et dénigrements - souvent subis dans le cadre scolaire ou amical, rappelons-le. Ceux dont la mère était au foyer ont au contraire subi plus de violences physiques. Il semble aussi que les hommes qui n'avaient ni demifrères ni demi-sœurs aient été deux fois moins souvent brutalisés que les autres. A l'inverse, les femmes qui, à quatorze ans, ne vivaient ni avec leur père ni avec leur mère ont été deux fois plus nombreuses que les autres à déclarer avoir subi dans leur enfance ou leur adolescence des atteintes à leur dignité et des attouchements ou agressions sexuelles.

On décèle aussi un lien entre les violences subies avant dix-huit ans et l'histoire migratoire, en particulier pour les femmes : celles qui sont nées hors de Martinique (dans l'hexagone, les autres pays de la Caraïbe ou ailleurs) ont déclaré significativement plus souvent avoir subi des violences sexuelles que celles qui sont nées en Martinique et, parmi ces dernières, celles qui ont vécu au moins un an hors de Martinique en ont également déclaré davantage que celles qui n'ont jamais vécu hors du département. On peut certes imaginer que les natives de Martinique qui ne l'ont jamais quittée aient minimisé, consciemment ou non, les violences subies dans l'enfance. Mais il est également probable que les souffrances ressenties, et le besoin de mettre de la distance avec un contexte difficile, aient joué un rôle dans le parcours migratoire des répondantes - dans leur départ (avec ou sans leurs parents) de Martinique ou dans leur retour (seules ou avec leurs parents) en Martinique pour celles qui y sont nées, et dans leur venue en Martinique pour celles qui n'y sont pas nées.

Pour les femmes, la pratique religieuse actuelle semble aussi discriminante : celles qui se disent aujourd'hui «sans religion» et, plus encore, celles qui ont déclaré pratiquer une religion autre que catholique sont nettement plus nombreuses que celles qui se sont dites catholiques à avoir relaté des atteintes sexuelles et surtout des violences physiques. Toutefois, en l'absence d'indications sur les croyances et pratiques religieuses des familles des répondantes, on ne peut déterminer le sens de ce lien: les femmes issues de familles catholiques ont-elles été moins exposées que les autres dans leur jeunesse aux violences physiques et sexuelles ? Ou bien, les femmes qui ont subi de telles violences se sont-elles par la suite plus souvent détournées de toute pratique religieuse ou tournées vers d'autres églises ou croyances ? Quoiqu'il en soit, on peut rapprocher ce constat de celui fait dans les relations conjugales pour les douze derniers mois: on verra que l'absence de pratique religieuse expose un peu plus les femmes à des atteintes psychologiques, de niveau faible ou moyen, et que, à l'inverse, la pratique de la religion (catholique) semble les en protéger. 
D'autres liens statistiquement très significatifs avec les violences subies dans l'enfance ou l'adolescence sont plutôt à regarder comme des conséquences et non comme des causes de ces violences: comme pour tous les faits associés à une enfance difficile, on trouve ainsi un lien très fort, pour les deux sexes, entre la déclaration de violences subies avant dix-huit ans et celle de tentatives de suicide. Le lien est également fort, pour les hommes, et très fort, pour les femmes, entre le fait d'avoir rapporté des violences avant dix-huit ans, quel qu'en soit le type, et le sentiment constant de tension, stress ou déprime dans les semaines qui ont précédé la collecte ${ }^{3}$.

\section{III. - LES VIOLENCES SUBIES AU COURS DES DOUZE DERNIERS MOIS}

Il faut d'abord souligner que, quel que soit l'espace de vie, deux tiers des répondant(e)s ont déclaré n'avoir subi aucun fait de violence au cours des douze derniers mois. Dans tous les modules, les atteintes les plus fréquemment déclarées sont les atteintes à la dignité ou à la personnalité et les atteintes verbales. Sauf pour les atteintes sexuelles, les hommes sont un peu plus touchés que les femmes dans la vie professionnelle et dans l'espace public. Les femmes le sont davantage, surtout pour les faits les plus graves ou répétés, dans la vie conjugale, ainsi que dans les relations avec les proches ou avec un ancien conjoint ou partenaire. Les auteurs des faits déclarés sont plus souvent des hommes, tandis que les victimes, surtout pour les faits de violence physique ou sexuelle, sont plus souvent des femmes.

\section{A. - Dans l'espace public}

Un peu plus de $13 \%$ des femmes et $12 \%$ des hommes enquêtés ont déclaré avoir subi au moins une atteinte ou une agression dans l'espace ou dans un lieu publics au cours des douze derniers mois. Comme dans les autres sphères de la vie, ce sont les insultes qui sont les faits les plus souvent

\footnotetext{
${ }^{3}$ Un tiers des hommes et près de la moitié des femmes ont répondu qu'ils s'étaient sentis «constamment tendus, stressés ou déprimés» au cours des trois semaines précédant l'enquête. Le travail ou le chômage et l'argent sont avancés comme les causes principales de stress, suivies par la santé et, pour les femmes, par les problèmes familiaux. Ce sont les femmes nées en Martinique et qui n'ont jamais vécu ailleurs, les personnes qui ont moins de 1000 euros de revenu mensuel et/ou qui n'ont aucun diplôme et, plus encore, les femmes en situation monoparentale, qui ont été les plus nombreuses à se déclarer «constamment tendues, stressées ou déprimées ». 33 hommes et 69 femmes sur mille ont répondu avoir déjà fait une tentative de suicide, dont 12 hommes et 17 femmes sur mille plusieurs fois. Pour 6,6 hommes et 7,4 femmes sur mille, la dernière tentative s'est produite dans les douze mois précédant l'enquête. Les tentatives déclarées semblent avoir été plutôt le fait des personnes nées dans l'hexagone ou y ayant longtemps vécu.
} 
déclarés par les deux sexes (tableau 2), suivis, pour les femmes, par les propos ou gestes sexuels gênants ou obscènes. Les répondants ont le plus souvent considéré les uns et les autres comme « pas graves ».

Tableau 2 : Taux (en \%) d'atteintes et violences subies dans les espaces publics pendant les douze derniers mois, selon le sexe

\begin{tabular}{|l|c|c|}
\hline \multicolumn{1}{|c|}{ Type de violence déclarée } & Femmes & Hommes \\
\hline Insultes & 7,5 & 7,4 \\
\hline $\begin{array}{l}\text { Suivi(e) avec insistance, menaces de mort verbales } \\
\text { ou avec une arme }\end{array}$ & 4,4 & 3,3 \\
\hline $\begin{array}{l}\text { Agressions physiques (vol avec violence, gifles, } \\
\text { coups avec arme, tentative de meurtre) }\end{array}$ & 0,9 & 2,5 \\
\hline $\begin{array}{l}\text { Agressions sexuelles (attitudes et propos sexuels } \\
\text { gênants, choquants; rapports forcés ou tentatives de } \\
\text { rapports forcés*) }\end{array}$ & 5,7 & 2,6 \\
\hline $\begin{array}{l}* \text { une femme a déclaré un viol et trois hommes des tentatives de viol (2) ou/et } \\
\text { viols(2) }\end{array}$ & \\
\hline
\end{tabular}

Par contre, plus de la moitié de ceux et celles qui ont déclaré avoir été suivi(e)s de façon insistante ou menaçante (troisième fait déclaré), presque toujours par des hommes (et même assez souvent par plusieurs hommes), ont considéré qu'il s'agissait d'un fait grave.

Les autres faits (vols avec violences, brutalités physiques, menaces ou coups avec arme, tentative de meurtre) n'ont été déclarés que par $2 \%$ au plus des répondants. Ils n'ont pas toujours été regardés comme graves par les victimes, mais ont eu des conséquences sérieuses pour certaines: onze hommes et trois femmes ont été blessés, la plupart des hommes assez gravement.

Tous faits confondus, les femmes ne semblent donc qu'à peine plus souvent victimes d'agressions dans l'espace public que les hommes, et moins souvent d'agressions aux conséquences physiques graves. Sans doute parce qu'elles évitent de s'y retrouver seules, surtout le soir : les femmes ont presque toujours été agressées dans la journée, alors que la moitié des hommes l'ont été le soir ou surtout la nuit. Pour les hommes comme pour les femmes, la majorité des agressions se sont produites dans un lieu habituellement fréquenté, même si, pour les hommes, c'était un peu plus souvent dans des lieux inhabituels. Dans la moitié des cas d'agressions graves, la victime n'était pas seule avec son agresseur et, dans la moitié des cas également, quelqu'un est intervenu pour la défendre. Quatre hommes 
(sur 500) et onze femmes (sur 1000) ont porté plainte contre leurs agresseurs.

Les insultes sont proférées par un homme dans les trois quarts des cas lorsque l'insulté est également un homme, et dans un tiers des cas lorsqu'elles s'adressent à une femme. Les agressions commises par les femmes sont essentiellement verbales. Les menaces et agressions physiques sont par contre le fait des hommes, seuls ou en groupe : les neuf dixièmes des menaces et agressions physiques à l'égard des femmes sont le fait d'hommes seuls, tandis que les deux cinquièmes des agressions commises à l'égard des hommes le sont par des hommes en groupe. Dans un cas sur trois (et dans près d'un cas sur deux lorsqu'il s'agit d'agressions physiques), les hommes connaissaient l'auteur des agressions. Les femmes, au contraire, ne connaissaient que rarement leurs agresseurs : une fois sur sept environ.

\section{B. - Dans la vie professionnelle ou étudiante}

Au travail, pour les deux sexes, les atteintes les plus fréquemment déclarées sont les critiques injustes du travail effectué et le dénigrement, suivies des brimades et des insultes, et, pour les femmes, des propos ou comportements sexuels gênants ou choquants (tableau 3). Les autres faits ne touchent que moins de $4 \%$ des répondant(e)s : menaces de mort, surtout déclarées par des hommes, violences physiques et, enfin, agressions sexuelles, déclarées par $0,4 \%$ des femmes. Il faut souligner que 5,6\% des hommes et 6,1\% des femmes ont fait état de cumul, «de répétition » ou de gravité des atteintes verbales ou morales que l'on peut dès lors considérer comme du harcèlement moral.

Tableau 3 : Taux (en \%) d'atteintes et violences subies dans les douze derniers mois au travail selon le sexe

\begin{tabular}{|l|c|c|}
\hline Type de violence déclarée & Femmes & Hommes \\
\hline Critiques injustes et dénigrement & 12,7 & 17,4 \\
\hline Brimades dans l'organisation du travail & 8,1 & 10,3 \\
\hline Destruction du travail ou de l'outil de travail & 1,6 & 1,9 \\
\hline Insultes & 7,1 & 8,7 \\
\hline Menaces de mort verbales ou avec une arme & 1,6 & 3,2 \\
\hline Agressions physiques & 1,6 & 1,1 \\
\hline Propos et attitudes sexuels gênants, choquants & 4,9 & 1,6 \\
\hline Agressions sexuelles & 0,4 & -- \\
\hline
\end{tabular}


Un ou des hommes sont mis en cause dans les deux tiers des atteintes verbales et morales au travail. Qu'elles concernent un homme ou une femme, ces atteintes sont d'abord le fait des supérieurs hiérarchiques, des patrons ou des collègues, mais les subordonnés et usagers ou clients apparaissent plus agressifs envers les femmes. Les atteintes et agressions sexuelles au travail sont encore huit fois sur dix perpétrées par des supérieurs ou des collègues.

Les personnes ayant subi des brimades, des critiques injustes, du dénigrement ou des insultes qu'elles jugent graves se sont plaintes deux fois sur trois, d'abord auprès d'un supérieur hiérarchique, puis moins souvent auprès d'un représentant du personnel ou responsable syndical, plus rarement encore auprès de l'inspecteur du travail. A la suite de ces plaintes, les poursuites ou mesures prises à l'encontre des auteurs ont été rares, une fois sur six pour les brimades et critiques, une fois sur trois pour les insultes. Dix personnes (cinq hommes et cinq femmes) menacées verbalement de mort ont pris ces menaces au sérieux. Mais, dans l'ensemble, les répondant(e)s relativisent souvent l'importance des atteintes et violences subies dans l'exercice de leur profession. Ils n'en parlent pas ou, s'ils en parlent à leurs proches et à leur entourage professionnel, ils font peu de démarches à caractère judiciaire et les poursuites sont rares. Cependant, ils en subissent de nombreuses conséquences dans leur vie personnelle. Le fait que les femmes actives en exercice au moment de l'enquête aient très nettement moins souvent déclaré des atteintes et violences sexuelles que celles qui ont pris leur retraite ou sont au chômage depuis moins de douze mois conduit à penser que certaines de ces dernières ont pu quitter leur emploi pour échapper à ces faits.

Les 70 répondants et les 82 répondantes qui se considèrent principalement étudiant(e)s (même s'ils exercent par ailleurs un travail à temps partiel ou un djob) n'ont déclaré aucune atteinte grave dans le cadre de leurs études, à l'exception d'une femme victime de harcèlement sexuel.

\section{C. - Dans la famille et l'entourage}

La grande majorité des répondants, hommes et femmes, n'ont déclaré aucun fait commis à leur encontre par des membres de leur famille (autres que le conjoint) ou de celle de leur conjoint, par des amis, des proches ou des voisins, si ce n'est quelques injures, qui, d'ailleurs, deux fois sur trois, n'ont pas été considérées comme graves et qui, une fois sur deux, ne se sont pas répétées.

Mais, dans les cas, peu nombreux ( $1 \%$ des hommes et $1,3 \%$ des femmes), où les faits déclarés dépassent le niveau des injures bénignes, des 
mises à la porte ou des refus d'accueil et des menaces de mort non prises au sérieux, ces faits peuvent être graves : menaces de mort prises au sérieux, brutalités, menaces avec arme et tentatives de meurtre, viol. Les auteurs sont alors essentiellement des hommes : des amis pour les agressions envers les hommes, et des membres de la famille pour les agressions envers les femmes.

Les femmes ont été les seules à déclarer avoir été victimes d'atteintes et violences à caractère sexuel de la part de proches au cours des douze derniers mois : les auteurs désignés en sont des membres de la famille (et d'abord les frères) pour trois femmes sur quatre.

Enfin, sur les trente-six personnes qui ont jugé graves les insultes qui leur ont été adressées, neuf les ont jugées suffisamment graves pour porter plainte et des poursuites ont été engagées contre quatre des auteurs.

\section{D. - Dans le couple}

Les concepteurs des questionnaires Enveff ont considéré, rappelonsle, que les violences au sein des couples formaient un continuum incluant les agressions verbales, psychologiques, physiques et sexuelles et que, par-delà les actes violents caractérisés, comme les brutalités physiques et les agressions sexuelles, l'accumulation de faits, de gestes, de paroles en apparence sans grande gravité créait des situations pouvant porter gravement atteinte aux victimes. Ainsi, les questions posées aux personnes ayant déclaré avoir une relation de couple, cohabitant ou non, au moment de l'enquête, visaient à mesurer cinq types d'atteintes et violences conjugales :

- la contrainte économique (1 question);

- les atteintes à la dignité et les violences psychologiques (8 questions) ;

- les menaces en paroles ou avec armes (3 questions);

- les violences physiques (4 questions) ;

- les atteintes et violences sexuelles (4 questions).

Il faut d'abord souligner que la plupart des répondant(e)s n'ont déclaré aucun fait de violence, psychologique ou autre, de la part de leur partenaire. Ainsi, 53,5\% des femmes et $50 \%$ des hommes n'ont déclaré aucun fait ; $18 \%$ des premières et $20 \%$ des seconds n'ont rapporté qu'une ou deux atteintes seulement, non répétées et pas jugées graves, dans l'année. 
Tableau 4: Taux (en \%) d'atteintes et violences subies dans la relation conjugale pendant les douze derniers mois selon le sexe

\begin{tabular}{|l|c|c|}
\hline \multicolumn{1}{|c|}{ Type de violence déclarée } & Femmes & Hommes \\
\hline Atteintes à la dignité et violences psychologiques & 22,3 & 26,1 \\
Dont : manifestations de jalousie & 9,4 & 12,5 \\
critiques, mépris, insultes & 14,3 & 13,5 \\
\hline Contrainte économique & 6,7 & 2,2 \\
\hline Menaces en paroles ou avec arme & 2,0 & 2,1 \\
\hline Agressions physiques & 2,5 & 1,9 \\
\hline Atteintes et violences sexuelles & 7,6 & 4,4 \\
\hline
\end{tabular}

Cependant, environ un quart des personnes ayant une relation conjugale ont été soumises à des atteintes à leur dignité ou à des violences psychologiques pendant les douze derniers mois (tableau 4). Ce sont les critiques et le mépris, l'inquisition sur les fréquentations et le refus de discuter qui sont le plus souvent dénoncés, d'ailleurs autant par les hommes, qui se plaignent surtout que leur compagne exige de savoir où et avec qui ils étaient, que par les femmes qui placent les critiques et le mépris avant ce même reproche. Mais une femme sur dix fait état de cumuls et/ou de fréquences des faits qui confinent au harcèlement. Ces proportions sont proches de celles observées dans l'hexagone huit ans auparavant, mais un peu inférieures à celles qui ont été calculées pour l'île de La Réunion et très inférieures à celles observées en Polynésie française.

La privation d'argent pour les besoins de la vie quotidienne apparaît très variable selon le type de relation conjugale : 6,7 \% pour l'ensemble des femmes ayant une relation de couple et $5,5 \%$ pour celles qui vivent avec leur conjoint. Parmi les femmes qui ne vivent pas avec leur ami, $5 \%$ de celles qui n'ont pas d'enfant de lui, mais $22,6 \%$ des autres se plaignent de son insuffisance ou de son absence de contribution aux besoins quotidiens.

Viennent ensuite les pratiques sexuelles imposées, les tentatives de viol et les viols dénoncés par 7,6\% des femmes (dont 5,7\% de viols ou tentatives de viols $\left.{ }^{4}\right)$. Quant aux hommes qui déclarent avoir subi des atteintes sexuelles, plus de la moitié ne vivent pas avec leur compagne et la

\footnotetext{
${ }^{4}$ Appliqués à l'ensemble des femmes de 18 à 59 ans vivant en Martinique et entretenant une relation de couple, ces résultats signifient que 6000 femmes au moins ont, au cours de l'année écoulée, été victimes de tentatives de rapports forcés ou de rapports forcés par leur conjoint ou ami.
} 
moitié ont ou ont eu plusieurs partenaires sexuelles dans l'année; on peut ainsi supposer que ces atteintes réprouvées relevaient pour une large part de sollicitations de compagnes délaissées ou trompées tentant de «reprendre leur homme».

La fréquente symétrie apparente entre les proportions de «victimes » féminines et masculines, entre les atteintes déclarées par les hommes et celles déclarées par les femmes, pour ce qui concerne les atteintes à la dignité, les violences psychologiques et les menaces, voire les atteintes sexuelles, est en effet trompeuse. Tout d'abord, il n'est pas certain que des items adoptés pour mesurer des violences psychologiques s'inscrivant dans un continuum de violences conjugales et dans un registre de domination masculine puissent signifier la même chose pour les hommes et pour les femmes - et ce, sans doute encore moins dans un contexte caribéen, où l'injonction à la virilité (et à la « réputation» masculine) est aussi souvent une injonction au pluripartenariat masculin, tandis que la socialisation féminine encourage la «respectabilité » associée à la monogamie et à la fidélité 5 . Ainsi, au moins un tiers des hommes relatant la jalousie de leur conjointe ont ou ont eu plusieurs partenaires sexuelles dans l'année (contre une femme sur six environ dans la même situation). Et si l'on exclut les items portant sur le contrôle de leurs relations extra-conjugales, le pourcentage d'hommes qui atteignent le niveau élevé d'atteintes psychologiques déclarées (que l'on peut assimiler à du harcèlement moral) chute de $1,6 \%$ à $0,3 \%$.

Par ailleurs, si 2 à $3 \%$ des uns comme des autres ont déclaré avoir été victimes de faits considérés a priori comme graves (menaces de mort, confiscation de papiers, brutalités physiques, menaces ou coups avec arme, tentatives de meurtre, rapports sexuels forcés), un homme sur dix seulement, mais trois femmes sur quatre ont estimé qu'il s'agissait réellement de quelque chose de grave. Aucun homme n'a répondu avoir été blessé et aucun n'a fait une main courante ou porté plainte; dans aucun cas jugé grave, le fait n'a été réitéré. Au contraire, la moitié des faits jugés graves par les femmes ${ }^{6}$ se sont produits plusieurs fois, voire souvent, et, dans un cas sur deux, ils ont entraîné des blessures et/ou donné lieu à des mains courantes ou à des dépôts de plainte.

\footnotetext{
${ }^{5}$ Nadine Lefaucheur \& Stéphanie Mulot. «Pluripartenariat masculin et violences conjugales : les effets de l'injonction à la virilité sur les relations de couple en Martinique». Communication au colloque Les coûts de la masculinité, Rennes, 15-16 janvier 2010 (à paraître sous le titre «La construction et les coûts de l'injonction à la virilité en Martinique » dans les actes du colloque aux Presses universitaires de Rennes).

${ }^{6}$ Neuf des treize femmes victimes des faits de violence conjugale les plus graves ont accepté de rencontrer une enquêtrice en face à face. Cinq entretiens ont été réalisés (voir, dans ce numéro, la III ${ }^{\mathrm{e}}$ partie : « Sortir de la violence conjugale »).
} 
Enfin, il semble que les formulations : «votre conjoint(e) a essayé d'avoir un rapport sexuel avec vous contre votre volonté » et «votre conjoint(e) vous a forcé(e) à avoir un rapport sexuel avec lui/elle»adoptées pour éviter les connotations trop fortes du terme «viol»- ou la formulation: "votre conjoint(e) vous impose des pratiques sexuelles qui vous déplaisent»n'aient pas été (et ne puissent sans doute pas être) entendues de la même façon par les hommes et par les femmes. Lorsqu'elles ont déclaré avoir eu des rapports «contre leur volonté », les femmes les ont considérés comme des faits graves, obtenus par l'intimidation, les menaces ou la force physique, à un moment où elles parlaient de séparation ou étaient soupçonnées d'avoir d'autres relations, et toutes sauf une ont fait état de conséquences graves pour leur équilibre psychologique et leur vie conjugale ou amoureuse, voire leur vie sociale, leur santé et leur sexualité. Par contre, les hommes qui ont déclaré avoir eu des rapports contre leur volonté les ont imputés à un chantage et décrits comme «non graves» et sans conséquences. Les entretiens réalisés en face à face conduisent d'ailleurs à se demander si les pratiques sexuelles «déplaisantes» que des hommes reprochent à leurs partenaires ne se réfèrent pas au moins aussi souvent au manque de disponibilité et d'imagination érotique (ou pornographique) dont ces partenaires feraient preuve à leurs yeux qu'à des pratiques particulières qu'elles chercheraient à leur imposer - si ce n'est, parfois, l'abstinence ou le port du préservatif lorsqu'ils ont d'autres relations et sont, ou sont susceptibles d'être, contaminés par le VIH (virus du sida).

La comparaison des résultats obtenus en Martinique avec ceux des précédentes enquêtes Enveff ${ }^{7}$ concernant les violences conjugales dont les femmes ont déclaré avoir été victimes au cours de l'année écoulée montre que la proportion de celles qui ont subi au moins une agression physique est proche du chiffre de l'hexagone ou de La Réunion, mais très inférieure à celui de la Polynésie. La proportion de femmes ayant fait état de cumuls et/ou de fréquences des faits qui confinent au harcèlement psychologique (10\% des femmes en couple) est proche de celles qui ont été calculées pour l'hexagone ou pour La Réunion, mais très inférieure à celles relevées en Nouvelle-Calédonie et en Polynésie française (24\%). En revanche, la privation d'argent pour les besoins de la vie quotidienne a été beaucoup plus souvent déclarée en Martinique que dans l'hexagone : 6,8\% vs 1,2\%. Par ailleurs, à moins qu'elles n'en parlent plus facilement, les femmes vivant en Martinique subiraient beaucoup plus souvent des agressions sexuelles de la

\footnotetext{
${ }^{7}$ Elizabeth Brown \& Isabelle Widmer. «Violences envers les femmes en métropole et outremer : continuités et différences » in Natacha Chetcuti \& Maryse Jaspard (dir). Violences envers les femmes : trois pas en avant, deux pas en arrière. L'Harmattan, 2007, p. 223-239. Voir aussi, dans ce numéro: "Les enquêtes Enveff sur les violences envers les femmes dans la France hexagonale et ultramarine ».
} 
part de leur conjoint ou partenaire que celles qui vivent dans l'hexagone ou à La Réunion ( $7,6 \%$ vs $0,8 \%$ et $1,3 \%)$.

Comme cela avait été le cas dans les autres enquêtes Enveff, on constate en Martinique un cumul fréquent des types de violence «conjugale » déclarées par les femmes : parmi les $10 \%$ de femmes en couple soumises à un niveau élevé d'atteintes psychologiques (ou harcèlement moral), trois sur dix ont déclaré avoir subi des agressions sexuelles, trois sur dix également ont fait état du refus de leur partenaire ou conjoint de leur donner de l'argent pour les besoins de la vie quotidienne ; enfin, une sur dix a aussi rapporté des menaces ou des agressions physiques (répétées dans deux cas sur trois).

Les faits les plus graves déclarés par les femmes (menaces de mort, coups, tentatives de meurtre, rapports sexuels forcés) se sont parfois produits dès le début de la relation, mais le plus souvent à un moment où elles parlaient de séparation ou lorsque leur partenaire pensait qu'elles avaient rencontré quelqu'un d'autre.

Les femmes victimes de viol conjugal en avaient toutes parlé avant l'enquête, à leur mère ou à des proches, mais n'avaient entrepris aucune démarche judiciaire. Les autres agressions considérées comme graves ont été tues à l'entourage une fois sur deux. Mais, presque toutes les victimes évoquent des conséquences graves pour leur équilibre psychologique et leur vie conjugale ou amoureuse, voire leur vie sociale, leur santé et leur sexualité.

\section{E. - Dans les rapports avec un(e) ancien(ne) partenaire}

Un tiers des hommes et $21 \%$ des femmes ont eu des contacts avec d'anciens conjoints ou partenaires au cours de l'année écoulée : près de trois femmes et de deux hommes sur dix ont déclaré que ces contacts avaient été difficiles ou très difficiles.

Les insultes réitérées ont été, pour les deux sexes, les atteintes les plus fréquemment déclarées de la part d'un ancien conjoint ou partenaire (par une personne sur trois qui ont eu des contacts difficiles), mais, si moins d'un tiers des hommes les ont considérées au moins une fois comme graves, cela a été le cas de plus de quatre femmes sur cinq.

Viennent ensuite, pour une femme sur six et un homme sur dix, le harcèlement, à domicile, au travail, ou sur la voie publique.

Les brutalités déclarées ont été, comme cela était prévisible, plus souvent exercées par un homme à l'encontre de son ex-partenaire (un cas sur 
sept) que dans le sens inverse (un cas sur vingt); de plus, les victimes les ont considérées graves dans le premier cas et pas dans le second.

Notons encore que les menaces concernant les enfants (subies par environ une personne sur douze) sont conformes aux situations les plus courantes : les femmes menacent leur ancien partenaire de l'empêcher de les voir et les hommes menacent leur ancienne compagne de les en séparer.

Trois femmes n'avaient jamais parlé avant l'enquête, l'une des menaces de mort, les deux autres d'une tentative de viol ou d'un viol commis par leur ancien conjoint. Cependant, neuf femmes ont porté plainte et deux ont fait une main courante, pour insultes, harcèlement, menaces ou coups, et un homme a fait une main courante pour harcèlement.

\section{F. - Le cumul des risques et des violences}

De même que l'on a mis en évidence des cumuls de types de violence dans le cadre du couple, on constate des concomitances significatives entre les violences subies dans la relation conjugale et les violences subies dans les autres espaces de vie, sans que l'on puisse pour autant inférer d'une antériorité ou d'une causalité des unes par rapport aux autres.

Les femmes qui ont fait état de violences conjugales diverses, répétées ou graves, ont ainsi déclaré presque trois fois plus d'atteintes verbales et morales et sept fois plus d'atteintes et violences sexuelles dans le cadre professionnel que celles dont la relation conjugale est apparue sereine. Des facteurs d'accroissement des risques sont à l'évidence communs aux deux sphères de vie (cf. infra). Mais il semble également que la déstabilisation et le stress consécutifs aux mauvais traitements subis dans l'une des sphères engendrent un aspect de fragilité ou un comportement vulnérable susceptibles d'accroître l'agressivité du partenaire ou de l'entourage professionnel. Ce cumul des violences conjugales et au travail était déjà apparu patent dans l'enquête Enveff dans l'hexagone.

Les femmes qui ont rapporté des violences conjugales sont aussi plus susceptibles d'avoir déclaré des violences de la part de membres de leur famille ou de proches, plus susceptibles également d'avoir fait état de violences subies dans l'espace public.

Ces résultats mettent en lumière, une fois de plus, le cumul des désavantages et des handicaps sociaux et familiaux, lesquels, comme le montre l'analyse statistique des facteurs accroissant la probabilité d'être victime de violences (cf. infra), s'enracinent largement dans les difficultés sociales et familiales rencontrées dans l'enfance et l'adolescence. 


\section{IV. - LES VIOLENCES ANTÉRIEUREMENT SUBIES À L'ÂGE}

\section{ADULTE}

Par défaut de remémoration ou relativisation de l'importance de faits anciens ou par difficulté à situer la frontière de l'année précédant l'enquête (et, sans doute aussi, par un effet de lassitude au terme d'une interrogation d'une durée moyenne de quarante-cinq minutes), les atteintes et violences de tous ordres rapportées pour la vie adulte antérieure aux douze derniers mois apparaissent très sous-estimées ${ }^{8}$ par rapport aux taux obtenus pour ces douze mois, qui sont sans doute plus significatifs de la fréquence effective des violences interpersonnelles à la Martinique.

A l'âge adulte, les humiliations et le dénigrement systématique suffisamment choquants pour être remémorés et rapportés alors qu'ils se sont produits (souvent largement) avant les douze derniers mois affectent à peu près autant les hommes et les femmes $(4,5$ et $5,1 \%)$, mais les uns comme les autres en déclarent moins que dans l'enfance ou l'adolescence ou en ont été moins marqués. En revanche, si les filles ont déclaré un peu moins de violences physiques et de menaces de mort que les garçons au cours de leur enfance et de leur adolescence, il n'en va pas de même à l'âge adulte, où les femmes ont été nettement plus nombreuses que les hommes à déclarer en avoir été victimes : parmi les personnes qui ont déclaré avoir subi des brutalités au cours de leur vie, celles-ci leur ont été infligées après dixhuit ans pour un homme seulement sur dix, mais pour quatre femmes sur dix (avec des taux respectifs de 1 et $3,4 \%$ ).

Le cadre dans lequel ont été perpétrées les violences rapportées apparaît très différent selon le sexe de la victime. Pour les femmes, les atteintes à la dignité et à la liberté, les violences physiques et les menaces sont intervenues la plupart du temps dans les relations conjugales ou amoureuses. Pour les hommes, au contraire, les humiliations et dénigrements ont été majoritairement imposés par des supérieurs ou des collègues au travail et les violences physiques et menaces exercées par d'autres hommes dans des lieux publics, les victimes connaissant plus d'une fois sur deux leurs agresseurs.

Pour leur vie adulte antérieure aux douze derniers mois, 3,9\% des femmes ont relaté des atteintes et violences sexuelles. Les attouchements sexuels rapportés leur ont été souvent imposés dans le cadre d'une relation amoureuse ou conjugale, mais aussi au travail ou dans les études et dans une relation amicale ou de voisinage. Les tentatives de viol ont été le plus souvent imputées à un conjoint ou un ex-conjoint ou ami et nettement plus

\footnotetext{
${ }^{8}$ Voir, dans ce numéro : «De l'enquête statistique à l'enquête sur les sorties de la violence conjugale : constitution de l'échantillon et démarche d'enquête ».
} 
rarement à un collègue ou un supérieur hiérarchique. Deux viols sur trois ont été attribués au conjoint ou ami au moment des faits, alors qu'un sur dix l'a été à « un homme de la famille». Un viol sur cinq s'est produit dans un lieu public ou un «autre lieu», mais, même dans ce cas, trois fois sur quatre l'auteur était connu de sa victime.

\section{V. - LES FACTEURS ASSOCIÉS AUX VIOLENCES SUBIES À} L'ÂGE ADULTE

Des analyses de régression logistique ont permis de mettre en évidence les principaux facteurs qui accroissent la probabilité pour les personnes enquêtées, toutes choses égales par ailleurs, de s'être déclarées victimes de certains faits violents ${ }^{9}$. Bien que certaines caractéristiques associées aux violences rapportées se retrouvent dans la plupart des configurations, ces facteurs varient cependant, au moins en importance, selon les sphères et les périodes de la vie adulte (année écoulée, ensemble des années précédentes). La taille plus réduite de l'échantillon masculin limite le nombre de facteurs identifiables pour les hommes.

\section{A. - Dans l'espace public}

Les facteurs qui accroissent la probabilité d'avoir subi des violences dans l'espace public au cours de l'année écoulée sont, toutes choses égales par ailleurs, pour les hommes :

- l'âge, pour les insultes et violences physiques (moins de 25 ans), et pour les menaces (moins de 35 ans);

- la situation dans l'enfance et l'adolescence, pour les menaces et violences physiques (au moins deux éléments de situation sociale ou familiale difficile) ;

- l'emploi ou la profession, pour les insultes (travailleur indépendant, en emploi précaire, et, surtout, fonctionnaire).

Pour les femmes, toutes choses égales par ailleurs, les facteurs d'accroissement des risques sont :

- l'âge, pour les menaces (moins de 25 ans), les insultes, les violences sexuelles et l'indicateur global de violences (moins de 35 ans);

\footnotetext{
${ }^{9}$ Les résultats de ces régressions (les coefficients applicables aux risques) sont détaillés dans les tableaux en annexe de ce chapitre.
} 
- l'origine ou le parcours migratoire, pour les menaces et violences sexuelles (être née ou avoir vécu ailleurs qu'en Martinique) ;

- les difficultés de l'enfance et de l'adolescence, pour les insultes, menaces, les violences physiques et sexuelles (au moins deux éléments de situation sociale ou familiale difficile) ;

- le type de relation conjugale, pour l'indicateur global de violences (non cohabitation);

- l'emploi ou la profession, pour les insultes (profession libérale, cadre supérieur ou étudiante).

Bien que l'enquête ne permette pas de vérifier cette hypothèse, ces facteurs sont vraisemblablement liés à des habitudes différentes de fréquentation de l'espace public selon l'âge, la biographie, le parcours migratoire, le mode de vie conjugal et le statut d'activité.

\section{B. - Dans la vie professionnelle ou étudiante}

Pour les hommes, c'est essentiellement le risque de menaces verbales ou avec un objet dangereux qui augmente, toutes choses égales par ailleurs, avec le nombre des difficultés éprouvées dans l'enfance ou l'adolescence, avec le fait d'avoir moins de vingt-cinq ans ou encore avec le travail de nuit. Cependant, les difficultés vécues dans la jeunesse amplifient aussi le risque d'atteintes verbales et morales.

Pour les femmes, le fait d'avoir vécu des difficultés dans l'enfance ou l'adolescence accroît les risques de subir des atteintes verbales et morales ou des atteintes et agressions sexuelles. Un âge inférieur à 25 ans augmente la probabilité de menaces, alors qu'entre 25 et 34 ans, c'est le risque d'atteintes sexuelles qui s'élève, de même que celui d'atteintes verbales ou morales entre 35 et 44 ans. Une expérience migratoire d'au minimum un an accroît le risque d'atteintes et violences sexuelles pour les natives de Martinique. Etre fonctionnaire entraîne un risque d'atteintes verbales et morales plus important et le travail de nuit amplifie le risque d'atteintes verbales et morales lorsqu'il est régulier, et celui de subir des menaces lorsqu'il est occasionnel. 


\section{C. - Dans les relations de couple}

Si l'on considère que, pour un homme, les inquisitions et les marques de jalousie de sa compagne constituent des violences psychologiques, les facteurs qui accroissent sensiblement le risque de subir de telles violences sont, toutes choses égales par ailleurs : le fait de ne pas habiter avec son amie, d'avoir déclaré des sujets de disputes (surtout plus de deux) dans le couple, d'avoir cité au moins deux difficultés vécues pendant la jeunesse, et l'entrée très précoce ou, au contraire, tardive dans la vie sexuelle active. Par ailleurs, les hommes qui ont eu plusieurs partenaires sexuelles au cours de l'année ont également encouru un risque accru de faire l'objet de menaces, surtout s'il s'agissait de relations menées en parallèle.

Pour les femmes, les facteurs augmentant le risque de subir des violences conjugales sont :

- le nombre déclaré de sujets de disputes dans le couple, qui accroît d'autant plus la probabilité de rapporter des violences conjugales de tout type qu'il est élevé ;

- les difficultés connues dans l'enfance, et particulièrement la fréquence des punitions injustes, des coups et de la maltraitance, qui accroissent la probabilité de subir des atteintes psychologiques et sexuelles ;

- l'infidélité connue ou supposée du partenaire, qui accroît la probabilité de subir tous les types de violences ;

- le fait d'avoir eu plus d'un partenaire au cours de l'année écoulée, qui accroît le risque de violences sexuelles de la part du conjoint ou partenaire principal ;

- un âge inférieur à 35 ans (pour les atteintes psychologiques et marques de jalousie) ou supérieur à 45 ans (pour les rapports sexuels forcés et autres contraintes sexuelles) ;

- enfin, pour les natives de Martinique, le fait d'avoir vécu une expérience migratoire accroît la déclaration d'atteintes psychologiques.

Pour les femmes qui vivent avec leur conjoint, le partage des tâches ménagères - indicateur de relations plus égalitaires - réduit la probabilité de rapporter des violences conjugales.

Pour celles qui ne vivent pas avec leur partenaire, et qui ont au moins un enfant de lui, la probabilité de subir des violences économiques est élevée : près d'une sur quatre (contre une sur quinze femmes qui vivent avec leur conjoint) s'est vu refuser de l'argent pour les besoins de la vie quotidienne. Mais, ce sont les femmes qui savent ou se doutent que leur 
partenaire est marié, vit habituellement avec une autre femme ou a d'autres partenaires, qui ont les probabilités les plus élevées de se voir refuser une contribution aux besoins de la vie quotidienne et de l'éducation de leurs enfants communs.

Le statut d'activité ou le revenu personnel des conjoints ou partenaires, le nombre d'enfants dans le ménage et leur lien de parenté avec chacun des conjoints ou partenaires n'ont pas une influence significative par rapport aux autres facteurs de variation des risques de violence conjugale.

\section{D. - Dans les relations avec la famille et les proches}

Avoir connu des difficultés d'ordre familial pendant l'enfance et l'adolescence accroît la probabilité d'en rencontrer à l'âge adulte pour les personnes que cela n'a pas amenées à «rompre les ponts » avec leurs proches ou avec ceux qui étaient à l'origine de ces difficultés. Ainsi, toutes choses égales par ailleurs, le risque de subir des violences dans le cadre des relations avec la famille ou les proches est nettement plus élevé pour les hommes et les femmes qui ont répondu positivement à au moins deux des questions portant sur les situations difficiles rencontrées dans leur jeunesse.

Par ailleurs, les femmes dont les parents étaient séparés ou divorcés lorsqu'elles étaient adolescentes et les personnes qui ont des demi-frères ou des demi-sœurs courent un risque plus élevé de rapporter des violences de la part des proches.

Les insultes et autres atteintes deviennent plus probables de la part des proches, toutes choses égales par ailleurs, quand les femmes résident avec ou à proximité de membres de leur famille ou de celle de leur conjoint, et, également lorsqu'elles ont répondu avoir «souvent pensé » qu'elles avaient « bu avec excès ».

Mais, pour les femmes, l'accroissement de la probabilité de subir des atteintes de la part de leurs proches est encore plus élevé toutes choses égales par ailleurs lorsqu'elles entretiennent une relation de couple empreinte de violences - cet effet dépassant ceux de la séparation de leurs parents, de l'existence de demi-frères ou demi-sœurs et des excès de boisson reconnus.

Enfin, les hommes en couple qui exercent un métier indépendant ou ceux qui n'ont pas travaillé toute l'année écoulée seraient aussi davantage agressés par leurs proches. 


\section{E. - Dans tous les espaces, avant la dernière année}

Pour ce qui concerne les violences subies après dix-huit ans, mais avant les douze derniers mois, étant donné le nombre réduit de déclarations de violences subies par les hommes, aucun calcul de régression n'a pu mettre en évidence, pour eux, de facteurs significativement associés aux violences déclarées.

Pour les femmes, ce sont les difficultés connues dans l'enfance qui accroissent le plus la probabilité de déclarer des violences subies à l'âge adulte (hormis les douze derniers mois) dans différentes sphères de la vie, qu'il s'agisse d'atteintes à la dignité, de menaces, de violences physiques ou sexuelles. L'histoire migratoire est également associée à la probabilité de déclarer des violences physiques (pour les non natives) ou sexuelles (pour les non natives ou pour les natives ayant vécu hors de Martinique).

\section{F. - Toutes époques et toutes sphères de la vie confondues}

Toutes époques et sphères de la vie confondues, ce sont les difficultés sociales et familiales rencontrées dans l'enfance et l'adolescence (misère, alcoolisme, violence, maltraitance, manque d'affection, etc.) qui sont l'un des principaux facteurs d'accroissement des risques de victimation pour chaque type de violences.

Le jeune âge des répondant(e)s apparait également lié à une probabilité plus élevée de subir des violences dans toutes les sphères, à l'exception des relations familiales et amicales.

La plupart des autres facteurs n'ont été mis en évidence que pour les femmes, soit qu'ils n'accroissent pas la probabilité pour les hommes d'être victimes, soit que la taille limitée de l'échantillon masculin n'ait pas permis de les faire apparaître. Outre le travail de nuit et l'appartenance à la fonction publique dans la sphère professionnelle, il s'agit essentiellement de l'origine et de l'histoire migratoire qui, pour la vie adulte ou l'année écoulée comme pour l'enfance et l'adolescence, sont corrélées aux atteintes sexuelles subies par les femmes, sans qu'il soit toujours possible de déterminer le sens de la relation entre violences subies et parcours migratoire. Les natives de l'île qui ne l'ont jamais quittée apparaissent ainsi plus protégées, les autres caractéristiques restant identiques, des atteintes sexuelles dans l'espace public et la vie professionnelle que celles qui ont vécu quelque temps, voire plusieurs années, ailleurs. Les femmes nées hors de Martinique subiraient plus de menaces dans l'espace public, ainsi que, pour l'âge adulte, d'agressions physiques. 
D'autres facteurs ne sont associés à une plus forte probabilité d'être victime de violences que dans certaines sphères de l'existence. Pour les hommes, il s'agit, dans les relations avec la famille ou les proches, de l'absence de relation de couple, du statut d'indépendant ou de la perte récente d'emploi, et, dans l'espace public, du statut d'indépendant ou de fonctionnaire ou de la précarité de l'emploi. Pour les femmes, il s'agit de la cohabitation intergénérationnelle, dans les relations avec la famille ou les proches, de l'emploi dans la fonction publique et du travail de nuit, dans la sphère professionnelle, et du statut de profession libérale, de cadre supérieur ou moyen, ou d'étudiante, dans l'espace public.

Mais, c'est surtout dans la sphère conjugale que l'on a pu constater l'influence de facteurs particuliers. Les deux principaux sont, pour chaque sexe, le nombre de motifs de dispute dans le couple et, pour les femmes, le fait d'avoir eu plusieurs partenaires au cours de l'année ou la certitude ou le soupçon que leur conjoint ou partenaire avait un autre foyer ou d'autres «amies ». Les femmes, peu nombreuses ( $4 \%$ des femmes ayant une relation de couple, avec ou sans cohabitation), qui ont déclaré plus d'un partenaire sexuel au cours de l'année écoulée ont aussi été exposées à un risque très supérieur de faire l'objet de critiques méprisantes et de refus de communication de la part de leur partenaire principal ou actuel, mais, surtout, d'agressions sexuelles (pratiques imposées, tentatives de viol et viols).

Celles qui savent ou supposent que leur partenaire vit avec une autre femme ou a d'autres partenaires (et qui sont elles-mêmes deux à trois fois plus souvent célibataires et/ou en situation monoparentale, après avoir vécu avec une mère seule, et plus souvent aussi que les autres, ouvrières, employées ou en emploi précaire) ont significativement plus souvent déclaré avoir été victimes de manifestations de mépris, d'insultes, de remarques désagréables, de refus de communication, de contrôle de leurs propres activités ou rencontres, de gestes sexuels gênants en public (qu'elles sont pratiquement les seules à avoir dénoncés), de pratiques sexuelles imposées et de tentatives de rapports forcés, de coups et brutalités et de menaces de mort, avec ou sans arme (menaces qu'elles sont les seules à avoir rapportées). Elles sont aussi près de quatre fois plus nombreuses à avoir essuyé des refus de participation aux besoins de la vie quotidienne et de l'éducation des enfants.

$\mathrm{Au}$ regard des analyses «toutes choses égales par ailleurs », les cumuls de violences entre différentes sphères de vie se confirment. Outre l'influence déjà mentionnée de l'existence de violences conjugales sur le comportement plus agressif de la famille et des proches, lorsque les régressions logistiques sont appliquées aux personnes ayant une relation de couple et exerçant ou ayant exercé un emploi dans l'année, on constate 
que les femmes qui ont fait état d'atteintes ou de violences de la part de leur conjoint ou partenaire ont couru, à caractéristiques identiques, un risque deux fois plus élevé de subir des atteintes verbales ou morales au travail, cet effet supplantant ceux de l'âge et du statut de fonctionnaire, alors que l'influence des situations de jeunesse difficiles reste prépondérant. On constate aussi que les femmes qui subissent des atteintes et violences au travail ont, à caractéristiques identiques, un risque deux fois et demi plus élevé de subir des violences psychologiques dans leur relation conjugale.

On peut supposer que c'est la taille plus limitée de l'échantillon masculin qui n'a pas permis de mettre en évidence de tels cumuls de violences pour les hommes. Mais, pour le sexe féminin, il y a bien un effet négatif, propre et réciproque, des atteintes dans la vie professionnelle sur la qualité de la relation conjugale. 
ANNEXE : RÉSULTATS DES RÉGRESSIONS LOGISTIQUES SUR LES FACTEURS EXPLICATIFS DES RISQUES DE VIOLENCES PAR SPHÈRE DE VIE

Pour chaque sphère, les facteurs potentiels de variation des risques introduits dans les régressions sont les caractéristiques des personnes qui sont apparues significativement liées aux fréquences observées des indicateurs de violences.

\section{A. - Violences dans l'espace ou les lieux publics}

Risque de référence: la personne de référence, femme ou homme, est âgée de 35 à 44 ans, elle est née en Martinique et n'a jamais vécu ailleurs. Elle vit avec son ou sa partenaire, a relaté au plus une difficulté dans la jeunesse, est employée en CDI et ne travaille jamais entre 21 heures et 6 heures du matin.

\begin{tabular}{|l|c|}
\hline \multicolumn{1}{|c|}{$\begin{array}{c}\text { Risque global de violence dans l'espace ou les lieux } \\
\text { publics dans les 12 mois }\end{array}$} & $\begin{array}{c}\text { Coefficient } \\
\text { d'accroissement du } \\
\text { risque (odds ratio) }\end{array}$ \\
\hline Femmes (risque de référence 0,025) & $2,5^{*}$ \\
\hline Âge à l'enquête : $18-24$ ans (vs 35-44 ans) & $2,3^{* *}$ \\
\hline Âge à l'enquête : 25-34 ans (vs 35-44 ans) & 1,6 \\
\hline $\begin{array}{l}\text { Ne vit pas avec son conjoint ou partenaire (vs en couple } \\
\text { cohabitant) }\end{array}$ & $2,4^{* *}$ \\
\hline Non native (vs native n'ayant jamais vécu ailleurs) & $2,0^{*}$ \\
\hline Native ayant vécu ailleurs (vs jamais vécu ailleurs) & $4,1^{* *}$ \\
\hline Nombre de difficultés dans l'enfance $: 2$ et plus (vs 0 ou 1) & 1,9 \\
\hline Cadre moyen (vs employée) & 2,2 \\
\hline Etudiante (vs employée) & 0,5 \\
\hline Ouvrière, agent ou personnel de service (vs employée) & 3,2 \\
\hline Hommes (risque de référence 0,023) & $3,8^{* *}$ \\
\hline Âge à l'enquête : $18-24$ ans (vs 35-44 ans) & $6,4^{*}$ \\
\hline Nombre de difficultés dans l'enfance : 2 et plus (vs 0 ou 1) & $5,8^{*}$ \\
\hline CDD ou emploi précaire (vs CDI) & $1,5^{*}$ \\
\hline Fonctionnaire (vs CDI) & $0,1^{*}$ \\
\hline Travailleur indépendant (vs CDI) & $0,3^{*}$ \\
\hline Cadre moyen (vs employé) & \\
\hline Ouvrier, agent ou personnel de service (vs employé) & \\
\hline $\begin{array}{l}\text { Le degré de significativité pour chaque facteur est inférieur à } 1 \%\left(^{* *}\right), 5 \% \text { ou } \\
\text { 10 \%. }\end{array}$ \\
\hline
\end{tabular}




\begin{tabular}{|l|c|}
\hline $\begin{array}{c}\text { Risque d'insultes dans l'espace ou les lieux publics dans } \\
\text { les 12 mois }\end{array}$ & $\begin{array}{c}\text { Coefficient } \\
\text { d'accroissement du } \\
\text { risque (odds ratio) }\end{array}$ \\
\hline Femmes (risque de référence 0,030) & $2,9^{* *}$ \\
\hline Âge à l'enquête : 25-34 ans (vs 35-44 ans) & $0,3^{*}$ \\
\hline Jamais vécu en couple (vs en couple cohabitant) & $4,3^{* *}$ \\
\hline Nombre de difficultés dans l'enfance : 2 et plus (vs 0 ou 1) & 2,4 \\
\hline Cadre supérieur ou profession libérale (vs employée) & $3,7^{*}$ \\
\hline Etudiante (vs employée) & 2,7 \\
\hline Hommes (risque de référence 0,028) & 3,8 \\
\hline Âge à l'enquête : $18-24$ ans (vs 35-44 ans) & $6,3 * *$ \\
\hline CDD ou emploi précaire (vs CDI) & 4,0 \\
\hline Fonctionnaire (vs CDI) & \\
\hline Travailleur indépendant (vs CDI) & \\
\hline $\begin{array}{l}\text { Le degré de significativité pour chaque facteur est inférieur à } 1 \%\left(^{* *}\right), 5 \% \text { ou } \\
10 \% \text {. }\end{array}$ \\
\hline
\end{tabular}

\begin{tabular}{|l|c|}
\hline $\begin{array}{c}\text { Risque de menaces verbales ou avec une arme dans } \\
\text { l'espace ou les lieux publics dans les 12 mois }\end{array}$ & $\begin{array}{c}\text { Coefficient } \\
\text { d'accroissement du } \\
\text { risque (odds ratio) }\end{array}$ \\
\hline Femmes (risque de référence 0,012) & $4,6^{* *}$ \\
\hline Âge à l'enquête : $18-24$ ans (vs 35-44 ans) & $2,0^{*}$ \\
\hline Nombre de difficultés dans l'enfance : 2 et plus (vs 0 ou 1) & $3,8^{*}$ \\
\hline Non native (vs native n'ayant jamais vécu ailleurs) & $2,4^{* *}$ \\
\hline $\begin{array}{l}\text { Native ayant vécu ailleurs (vs n'ayant jamais vécu } \\
\text { ailleurs) }\end{array}$ & \\
\hline Hommes (risque de référence 0,004) & $19,9^{* *}$ \\
\hline Âge à l'enquête : $18-24$ ans (vs 35-44 ans) & 7,2 \\
\hline Âge à l'enquête : 25-34 ans (vs 35-44 ans) & $4,2^{* *}$ \\
\hline Nombre de difficultés dans l'enfance : 2 et plus (vs 0 ou 1) & \\
\hline $\begin{array}{l}\text { Le degré de significativité pour chaque facteur est inférieur à } 1 \%\left(^{* *}\right), 5 \%\left(^{*}\right) \text { ou } \\
10 \% \text {. }\end{array}$ \\
\hline
\end{tabular}

\begin{tabular}{|l|c|}
\hline $\begin{array}{c}\text { Risque de violences sexuelles dans l'espace ou les lieux } \\
\text { publics dans les 12 mois }\end{array}$ & $\begin{array}{c}\text { Coefficient } \\
\text { d'accroissement du } \\
\text { risque (odds ratio) }\end{array}$ \\
\hline Femmes (risque de référence 0,010) & $8,2^{* *}$ \\
\hline Âge à l'enquête : $18-24$ ans (vs 35-44 ans) & $4,3^{* *}$ \\
\hline Âge à l'enquête : $25-29$ ans (vs 35-44 ans) & $3,5^{* *}$ \\
\hline Nombre de difficultés dans l'enfance : 2 et plus (vs 0 ou 1) & $2,9^{* *}$ \\
\hline Native ayant vécu ailleurs (vs n'ayant jamais vécu ailleurs) & \multicolumn{2}{|l}{} \\
\hline Hommes : aucun effet significatif & \\
\hline $\begin{array}{l}\text { Le degré de significativité pour chaque facteur est inférieur à } 1 \%(* *), 5 \%(*) \text { ou } \\
10 \% \text {. }\end{array}$ \\
\hline
\end{tabular}




\section{B. - Violences au travail ${ }^{10}$}

Risque de référence : la personne de référence, femme ou homme, a de 45 à 59 ans, est née en Martinique et n'a jamais vécu ailleurs. Elle a relaté au plus une difficulté dans la jeunesse, est ouvrière ou personnel de service, en CDI, travaille plus de 20 heures par semaine et jamais entre 21 heures et 6 heures du matin.

\begin{tabular}{|l|c|}
\hline $\begin{array}{c}\text { Risque d'atteintes verbales ou morales au travail dans } \\
\text { les } 12 \text { mois }\end{array}$ & $\begin{array}{c}\text { Coefficient } \\
\text { d'accroissement du } \\
\text { risque (odds ratio) }\end{array}$ \\
\hline Femmes (risque de référence 0,096) & $2,2^{* *}$ \\
\hline Âge à l'enquête : 35-44 ans (vs 45-59) & $3,2^{* *}$ \\
\hline Nombre de difficultés dans l'enfance : 2 et plus (vs 0 ou 1) & $2,1^{* *}$ \\
\hline Fonctionnaire (vs en CDI) & 0,6 \\
\hline $\begin{array}{l}\text { Travaille moins de 20 heures par semaine (vs plus de } 20 \\
\text { heures) }\end{array}$ & $2,2^{* *}$ \\
\hline Hommes (risque de référence 0,291) & Nombre de difficultés dans l'enfance : et plus (vs 0 ou 1) \\
\hline $\begin{array}{l}\text { Le degré de significativité pour chaque facteur est inférieur à } 1 \%(* *), 5 \%(*) \text { ou } \\
10 \% \text {. }\end{array}$ \\
\hline
\end{tabular}

\begin{tabular}{|l|c|}
\hline \multicolumn{1}{|c|}{$\begin{array}{c}\text { Risque de menaces verbales ou avec une arme au } \\
\text { travail dans les 12 mois }\end{array}$} & $\begin{array}{c}\text { Coefficient } \\
\text { d'accroissement du } \\
\text { risque (odds ratio) }\end{array}$ \\
\hline Femmes (risque de référence infime) & \\
\hline Hommes (risque de référence 0,003) & $11,0^{*}$ \\
\hline Âge à l'enquête $: 18-24$ ans (vs $45-59)$ & $13,9^{* *}$ \\
\hline Nombre de difficultés dans l'enfance $: 2$ et plus (vs 0 ou 1$)$ & $4,5^{*}$ \\
\hline Travaille occasionnellement de nuit (vs jamais) & \\
\hline $\begin{array}{l}\text { Le degré de significativité pour chaque facteur est inférieur à } 1 \%\left(^{* *}\right), 5 \%(*) \text { ou } \\
10 \% \text {. }\end{array}$ \\
\hline
\end{tabular}

\footnotetext{
${ }^{10}$ Les personnes qui se sont déclarées principalement étudiantes ont été exclues de ces analyses, en raison de leur effectif réduit, du faible nombre d'atteintes déclarées et de l'impossibilité d'assimiler leur cadre d'activité à celui des personnes exerçant un emploi. Pour les variations des risques de violences physiques au travail, aucun facteur ayant une influence significative n'a pu être dégagé.
} 


\begin{tabular}{|l|c|}
\hline $\begin{array}{c}\text { Risque d'atteintes et violences sexuelles au travail dans } \\
\text { les } 12 \text { mois }\end{array}$ & $\begin{array}{c}\text { Coefficient } \\
\text { d'accroissement du } \\
\text { risque (odds ratio) }\end{array}$ \\
\hline Femmes (risque de référence 0,009) & $4,0^{*}$ \\
\hline Âge à l'enquête : 25-34 ans (vs 45-59) & $4,1^{* *}$ \\
\hline Nombre de difficultés dans l'enfance : 2 et plus (vs 0 ou 1) & $2,6^{* *}$ \\
\hline $\begin{array}{l}\text { Native ayant vécu ailleurs (vs n'ayant jamais vécu } \\
\text { ailleurs) }\end{array}$ & $3,2^{*}$ \\
\hline Travaille régulièrement de nuit (vs jamais) & \\
\hline Hommes : aucun effet significatif & \\
\hline $\begin{array}{l}\text { Le degré de significativité pour chaque facteur est inférieur à } 1 \%\left(^{* *}\right), 5 \%(*) \text { ou } \\
10 \% \text {. }\end{array}$ \\
\hline
\end{tabular}

\section{C. - Relation conjugale ${ }^{11}$}

Risque de référence: la personne de référence, femme ou homme, est âgée de 35 à 44 ans, vit avec son partenaire, sans enfant au foyer. Elle exerce un emploi, a un revenu personnel inférieur à 1000 euros, a relaté au plus une difficulté dans la jeunesse, aucun sujet de dispute avec son/sa partenaire, a eu son premier rapport sexuel à 18 ou 19 ans, de deux à quatre partenaires sexuels dans sa vie, mais aucun autre partenaire dans l'année que celui avec lequel elle vit, ne croit pas que son/sa partenaire en ait eu. Elle est née en Martinique et n'a jamais vécu ailleurs.

\footnotetext{
${ }^{11}$ Pour les variations des risques de violences physiques perpétrées par le/la partenaire, aucun facteur ayant une influence significative n'a pu être dégagé.
} 


\begin{tabular}{|c|c|}
\hline Risque global de violence conjugale dans les 12 mois & $\begin{array}{c}\text { Coefficient } \\
\text { d'accroissement du } \\
\text { risque (odds ratio) }\end{array}$ \\
\hline \multicolumn{2}{|l|}{ Femmes (risque de référence 0,081 ) } \\
\hline Âge à l'enquête : $18-24$ ans (vs 35-44) & $2,4 * *$ \\
\hline Âge à l'enquête : 25-34 ans (vs 35-44) & $1,9 *$ \\
\hline Nombre de difficultés dans l'enfance : 2 et plus (vs 0 ou 1 ) & $3,1 * *$ \\
\hline Nombre de sujets de disputes : 1 ou 2 (vs 0 ) & $2,1 * *$ \\
\hline Nombre de sujets de disputes : 3 et plus (vs 0 ) & $3,9^{* *}$ \\
\hline Pluripartenariat du conjoint : sujet au doute (vs non) & $2,5^{* *}$ \\
\hline Pluripartenariat du conjoint : oui (vs non) & $2,2 * *$ \\
\hline Pluripartenariat de la femme : oui (vs non) & $4,0^{* *}$ \\
\hline Non native (vs native n'ayant jamais vécu ailleurs) & 0,6 \\
\hline \multicolumn{2}{|l|}{ Hommes (risque de référence 0,039 ) } \\
\hline Couple cohabitant : non (vs oui) & $3,0^{* *}$ \\
\hline Nombre de difficultés dans l'enfance : 2 et plus (vs 0 ou 1 ) & $2,9^{* *}$ \\
\hline Nombre de sujets de disputes : 1 ou 2 (vs 0 ) & 1,8 \\
\hline Nombre de sujets de disputes : 3 et plus (vs 0 ) & $3,3^{* *}$ \\
\hline Âge au premier rapport sexuel $:<=17$ (vs $18-19)$ & $3,0^{*}$ \\
\hline Âge au premier rapport sexuel : 20 et plus (vs 18-19) & $5,5^{* *}$ \\
\hline Pluripartenariat de la conjointe : oui (vs non) & $10,0^{*}$ \\
\hline \multicolumn{2}{|c|}{$\begin{array}{l}\text { Le degré de significativité pour chaque facteur est inférieur à } 1 \%(* *), 5 \%(*) \text { ol } \\
10 \% \text {. }\end{array}$} \\
\hline
\end{tabular}




\begin{tabular}{|c|c|}
\hline Atteintes psychologiques dans les 12 mois & $\begin{array}{c}\text { Coefficient } \\
\text { d'accroissement du } \\
\text { risque (odds ratio) }\end{array}$ \\
\hline \multicolumn{2}{|l|}{ Femmes (risque de référence 0,047 ) } \\
\hline Âge à l'enquête : 18-24 ans (vs 35-44 ans) & $3,7 * *$ \\
\hline Âge à l'enquête : 25-34 ans (vs 35-44 ans) & $2,3 * *$ \\
\hline Nombre de difficultés dans l'enfance : 2 et plus (vs 0 ou 1 ) & $3,1 * *$ \\
\hline Nombre de sujets de disputes : 1 ou 2 (vs 0 ) & $2,1^{*}$ \\
\hline Nombre de sujets de disputes : 3 et plus (vs 0 ) & $4,0^{* *}$ \\
\hline Pluripartenariat du conjoint : sujet au doute (vs non) & $1,9^{*}$ \\
\hline Pluripartenariat du conjoint : oui (vs non) & $2,3^{* *}$ \\
\hline Pluripartenariat de la femme : oui (vs non) & $4,1^{* *}$ \\
\hline Native ayant vécu ailleurs (vs jamais vécu ailleurs) & 1,6 \\
\hline \multicolumn{2}{|l|}{ Hommes (risque de référence 0,035) } \\
\hline Couple cohabitant : non (vs oui) & $3,1^{* *}$ \\
\hline Nombre de difficultés dans l'enfance : 2 et plus (vs 0 ou 1 ) & $2,4 * *$ \\
\hline Nombre de sujets de disputes : 1 ou 2 (vs 0 ) & $2,1^{*}$ \\
\hline Nombre de sujets de disputes : 3 et plus (vs 0 ) & $3,4^{* * *}$ \\
\hline Âge au premier rapport sexuel : <=17 (vs 18-19) & $3,0^{*}$ \\
\hline Âge au premier rapport sexuel : 20 et plus (vs $18-19$ ) & $5,3^{* *}$ \\
\hline
\end{tabular}

\begin{tabular}{|l|c|}
\hline \multicolumn{1}{|c|}{ Menaces verbales ou avec une arme dans les 12 mois } & $\begin{array}{c}\text { Coefficient } \\
\text { d'accroissement du } \\
\text { risque (odds ratio) }\end{array}$ \\
\hline Femmes : aucun effet significatif & \\
\hline Hommes (risque de référence 0,004) & 7,5 \\
\hline Pluripartenariat de l'homme : présumable (vs non) & $18,4^{*}$ \\
\hline Pluripartenariat de l'homme : oui (vs non) & Le degré de significativité pour chaque facteur est inférieur à 5\%(*) ou $10 \%$. \\
\hline
\end{tabular}




\begin{tabular}{|l|c|}
\hline \multicolumn{1}{|c|}{ Atteintes et violences sexuelles dans les 12 mois } & $\begin{array}{c}\text { Coefficient } \\
\text { d'accroissement du } \\
\text { risque (odds ratio) }\end{array}$ \\
\hline Femmes (risque de référence 0,009) & $2,8^{*}$ \\
\hline Âge à l'enquête : 45-59 ans (vs 35-44 ans) & $2,1^{*}$ \\
\hline Nombre de difficultés dans l'enfance : 2 et plus (vs 0 ou 1) & 2,3 \\
\hline Nombre de sujets de disputes : 1 ou 2 (vs 0) & $3,7^{* *}$ \\
\hline Nombre de sujets de disputes : 3 et plus (vs 0) & 2,2 \\
\hline Pluripartenariat du conjoint : sujet au doute (vs non) & $3,4^{* *}$ \\
\hline Pluripartenariat du conjoint : oui (vs non) & $6,3^{* *}$ \\
\hline Pluripartenariat de la femme : oui (vs non) & $11,5^{*}$ \\
\hline Hommes (risque de référence 0,028) & $5,5^{* *}$ \\
\hline Inactif (vs actif en emploi) & \\
\hline Pluripartenariat de l'homme : oui (vs non) & \\
\hline $\begin{array}{l}\text { Le degré de significativité pour chaque facteur est inférieur à } 1 \% \text { (**) }^{* *}, 5 \% \text { ou } \\
10 \% \text {. }\end{array}$ \\
\hline
\end{tabular}

\section{D. - Relations avec la famille et les amis}

Risque de référence: la personne de référence, femme ou homme, vivait avec ses deux parents à l'âge de quatorze ans, sans demi-frères ou demi-sours. Elle a relaté au plus une difficulté dans la jeunesse, vit avec son partenaire et des enfants, ne réside pas à proximité de sa famille ou de celle de son/sa partenaire. Elle est employée en CDI, a un revenu personnel compris entre 1000 et 2000 euros et n'a jamais pensé avoir trop bu pendant les douze derniers mois.

Rappelons que les atteintes déclarées dans ce cadre sont essentiellement des insultes et que l'analyse des variations du risque global est donc similaire à celle qui porterait seulement sur ces insultes. Les autres types de violences rapportés sont trop rares pour faire l'objet d'analyses particulières. 


\begin{tabular}{|c|c|}
\hline $\begin{array}{l}\text { Risque global de violence dans les relations familiales } \\
\text { ou amicales dans les } 12 \text { mois }\end{array}$ & $\begin{array}{c}\text { Coefficient } \\
\text { d'accroissement du } \\
\text { risque (odds ratio) }\end{array}$ \\
\hline \multicolumn{2}{|l|}{ Femmes (risque de référence 0,021 ) } \\
\hline $\begin{array}{l}\text { A } 14 \text { ans, avait des parents séparés (vs vivait avec ses } \\
\text { deux parents) }\end{array}$ & $2,2^{* *}$ \\
\hline A des demi-frères ou demi-sœurs (vs n’en a pas) & $1,7 *$ \\
\hline Nombre de difficultés dans l'enfance : 2 et plus (vs 0 ou 1 ) & $3,9^{* *}$ \\
\hline $\begin{array}{l}\text { Réside à proximité de sa famille ou de celle de son } \\
\text { partenaire (vs non) }\end{array}$ & $2,1 * *$ \\
\hline $\begin{array}{l}\text { A souvent pensé avoir trop bu pendant les } 12 \text { derniers } \\
\text { mois (vs jamais) }\end{array}$ & $9,0 *$ \\
\hline \multicolumn{2}{|l|}{ Hommes (risque de référence 0,004 ) } \\
\hline Nombre de difficultés dans l'enfance : 2 et plus (vs 0 ou 1 ) & $3,1 * *$ \\
\hline
\end{tabular}




\section{RÉSUMÉ}

Elizabeth Brown et Nadine Lefaucheur. Les violences subies dans les différents espaces de vie en Martinique

Sauf pour les atteintes sexuelles, les hommes sont un peu plus touchés que les femmes dans la vie professionnelle et dans l'espace public. Les femmes le sont davantage, surtout pour les faits les plus graves et répétés, dans la vie conjugale ou amoureuse, ainsi que dans les relations avec les proches ou avec un ancien conjoint ou partenaire. Les auteurs des faits déclarés par les hommes et par les femmes sont le plus souvent des hommes. Parmi les violences déclarées pour les périodes antérieures aux douze derniers mois, les plus nombreuses et les plus graves se sont produites pendant l'enfance et l'adolescence des répondants (humiliations, brutalités, attouchements). Toutes époques et sphères de la vie confondues, ce sont les difficultés sociales et familiales rencontrées dans l'enfance et l'adolescence - suivies par la jeunesse des répondants - qui sont l'un des principaux facteurs $\mathrm{d}$ 'accroissement des risques de victimation pour chaque type de violence (psychologique, physique, sexuelle). Dans la sphère amoureuse ou conjugale, et pour les douze derniers mois, s'ajoutent des facteurs spécifiques d'accroissement : le nombre de motifs de disputes dans le couple, ainsi que l'infidélité du conjoint victime ou, plus souvent, celle du conjoint violent.

\section{ABSTRACT \\ Elizabeth Brown and Nadine Lefaucheur. Violence experienced in different areas of life in Martinique}

Except for cases of sexual abuse, men in Martinique are slightly more affected by violence than women in both their workplaces and in public spaces. Women suffer violence more often, especially for the most serious and repeated incidents, in their romantic and familial relationships. In the cases of both male and female victims, they report that their aggressors are most often men. Among the incidents reported for periods prior to the last twelve months, the most numerous and most serious incidents occurred in childhood and adolescence (i.e. humiliations, beatings, inappropriate touching). Taking respondents of all ages and walks of life together, the main factors for increased risk of victimization for each type of violence (psychological, physical, sexual) were the social and familial difficulties encountered in childhood and adolescence. In the sphere of love and marriage, and for the last twelve months, certain factors contribute to higher rates of violence: the number of disputes between the members of the couple, as well as the infidelity of the victim, though more often, the infidelity of the violent partner. 